\title{
Energy loss by relativistic electron ensembles due to coherent excitation and ionization of atoms
}

\author{
S. V. Trofymenko $\oplus^{*}$ and N. F. Shul'ga \\ National Science Center Kharkiv Institute of Physics and Technology, \\ 1 Akademichna street, 61108 Kharkiv, Ukraine \\ and Karazin Kharkiv National University, 4 Svobody square, 61022 Kharkiv, Ukraine
}

(Received 7 May 2020; accepted 16 July 2020; published 13 August 2020)

\begin{abstract}
Coherent effects in the ionization loss of ultrarelativistic electron ensembles (bunches) are studied on the basis of perturbation theory of quantum mechanics. These effects originate from the interference of the electrons' proper electric fields with each other. General expressions for the ionization loss in this case, as well as their applicability conditions, are derived. It is shown that for ensembles of sufficiently small spatial size, achievable in modern accelerator facilities, the discussed effects lead to an increase of the ionization loss by several orders of magnitude compared to the result predicted by the Bethe-Bloch formula. In this case, the effective mean ionization potentials of the atomic shells depend not only on the structure of the atomic levels, but on the ensemble form factors as well. The numerical calculations are made for hydrogen atoms, taking into account the density effect in the ionization loss. The ionization loss due to resonant excitation and ionization of atoms by ensembles with periodical modulation of the particle density (realized at free-electron lasers) is investigated.
\end{abstract}

DOI: 10.1103/PhysRevAccelBeams.23.084501

\section{INTRODUCTION}

Interference effects in the ionization loss of charged particles have been studied previously for the case of microscopic groups of several particles. For instance, it was shown that the ionization loss of an ultrarelativistic electron-positron pair is reduced in the vicinity of the pair creation point in a substance, compared to the sum of independent losses by the electron and positron $[1,2]$. Such a reduction is caused by the destructive interference of the particles' electric fields with each other and is known as the Chudakov effect. Another example, concerning nonrelativistic particles, was studied in Refs. [3,4]. Here, the authors considered impinging of a cluster of several protons, bound by a common electron, on a solid target. Inside the target, such a cluster instantly lost the electron and suffered a "Coulomb explosion." In this case, immediately after the explosion the constructive interference between the protons' fields made the value of the particles' ionization loss several times larger than at the moment of time when the protons flew well apart from each other and the interference disappeared. The analogy between the

\footnotetext{
*trofymenko@kipt.kharkov.ua
}

Published by the American Physical Society under the terms of the Creative Commons Attribution 4.0 International license. Further distribution of this work must maintain attribution to the author(s) and the published article's title, journal citation, and DOI. processes considered in Refs. [1,2] and Refs. [3,4] was pointed out in Ref. [5].

In Ref. [6], analogous effects were considered for macroscopic ensembles of relativistic electrons consisting of $10^{5}-10^{9}$ particles with a total charge up to $1 \mathrm{nC}$. It was shown that, in this case, the interference effects can change the ionization loss much more significantly than in the case of microscopic groups of particles. Namely, for a sufficiently small size of such ensembles (bunches), their ionization loss can be coherently enhanced by several orders of magnitude. It resembles the phenomenon of coherent amplification of radiation by small electron bunches [7-9]. Bunches with the proper parameters required for the manifestation of the discussed effect are already obtained at modern x-ray freeelectron lasers and are going to be obtained at a series of accelerators presently under construction. This motivates the study of the interference effects in the ionization loss of such macroscopic particle ensembles. The application of such effects might be of interest for problems of the experimental diagnostics of the parameters of such ensembles.

In the discussed work [6], consideration of the ionization loss process was made in the framework of classical electrodynamics with the use of a simplified model of atomic electrons as classical harmonic oscillators. Such a model is rather nice for approximate estimations. Naturally, it did not allow accurately accounting for the electronic structure of the atoms excited or ionized by the impinging ensemble. However, the magnitude of the interference 
effects in the ionization loss is rather sensitive to the details of this structure and, particularly, to the values of the ionization potentials of the atomic shells.

In the present work, we develop a more accurate approach for the treatment of coherent effects in the ionization loss of macroscopic electron ensembles, based on perturbation theory of quantum mechanics. Such an approach allows obtaining general expressions for the effective values of the mean ionization potentials of the atomic shells which define the ensemble ionization loss. It is shown that, in general, such values depend on the ensemble form factors.

Let us also note that in Ref. [6] ionization loss in solid targets was studied. It was shown that, for the electron bunch parameters which are currently technically achievable, the discussed interference (coherent) effects can be manifested only in ultrathin targets, in which the density effect $[10,11]$ in the ionization loss is absent. In the present work, special attention is drawn to the gaseous targets, and the numerical results are presented for the simplest case of this kind which corresponds to atomic hydrogen. It is shown that in gases coherent effects can be manifested in the presence of the density effect as well and, therefore, for arbitrary values of the target thickness.

\section{CASE OF A SINGLE PARTICLE}

In the present work, we will consider the so-called restricted ionization loss. It is a part of the particle's mean ionization loss associated with the collisions accompanied by the momentum transfer less than some maximum value $\hbar \mathrm{q}_{0}$ (which corresponds to $\hbar \mathrm{q}_{1}$ in Ref. [12]). This quantity is often much more convenient for experimental measurements than the total loss due to collisions with arbitrary momentum transfer. If $\hbar \mathrm{q}_{0}$ is much less than the typical momentum of incident ultrarelativistic electrons from the ensemble, we can neglect the scattering of the latter ones. In this case, the velocity of the incident electrons is almost unchanged, and the total electromagnetic field created by the ensemble can be considered as an external perturbation of the atomic electrons' Hamiltonian. It triggers the transitions of these electrons to higher levels of the discrete (excitation) and continuous (ionization) spectrum.

Let us first consider the workability of this method for calculation of the ionization loss by a single particle in the absence of any coherent effects. In the nonrelativistic case, an analogous method was applied, for instance, in Refs. $[13,14]$ for the study of hydrogen atom ionization by multiply charged ions. We will presently consider the case of an ultrarelativistic incident electron taking into account the density effect, which can noticeably suppress the ionization loss at high particle energies.

As pointed out in Ref. [15], in order to accurately account for the incident external field in the Schrödinger equation for an atomic electron (with charge $e$ ), it is necessary to proceed from the relativistic Klein-Gordon equation $\left(\hat{\mathrm{p}}^{\mu} \hat{\mathrm{p}}_{\mu}-m^{2} c^{2}\right) \psi=0$ for the atomic electron wave function $\psi$ (we neglect any spin effects in our study). Making here the substitution $\hat{\mathrm{p}}^{\mu} \rightarrow \hat{\mathrm{p}}^{\mu}-e\left(A^{\mu}+a^{\mu}\right) / c$ and writing $\psi$ in the form $\psi=\phi(\mathbf{r}, t) e^{-i m c^{2} t / \hbar}$, in the nonrelativistic limit we obtain

$$
\begin{aligned}
i \hbar \frac{\partial \phi}{\partial t}= & \left(\frac{\hat{\mathbf{p}}^{2}}{2 m}+e \varphi_{n}+e A^{0}-\frac{e}{m c} \mathbf{A} \hat{\mathbf{p}}+\frac{e^{2}}{2 m c^{2}} \mathbf{A}^{2}\right) \phi \\
& +\frac{i e \hbar}{2 m c}\left(\frac{1}{c} \frac{\partial A^{0}}{\partial t}+\operatorname{div} \mathbf{A}\right) \phi .
\end{aligned}
$$

Here, $A^{\mu}=\left(A^{0}, \mathbf{A}\right)$ is the four-vector potential of the external field, while $a^{\mu}=\left(\varphi_{n}, 0\right)$ is the one of the atomic nucleus. Generally, $a^{\mu}$ describes the field of the nucleus together with the field of the rest of the atomic electrons, except the considered one, but this does not induce any changes in our present consideration.

The components of the four-vector potential of a relativistic incident electron with the velocity $\mathbf{v}$ in a polarized medium can be presented as follows [16]:

$$
\begin{aligned}
A^{0} & =e \frac{\exp \left\{-\frac{\omega_{p}}{c} \sqrt{\left(\boldsymbol{\rho}_{0}-\boldsymbol{\rho}\right)^{2}+\gamma^{2}(z-\mathrm{v} t)^{2}}\right\}}{\sqrt{\left(\boldsymbol{\rho}_{0}-\boldsymbol{\rho}\right)^{2} \gamma^{-2}+(z-\mathrm{v} t)^{2}}}, \\
\mathbf{A} & =\mathbf{v} A^{0} / c .
\end{aligned}
$$

The incident electron is assumed to move along the $z$ axis having radius vector $\rho_{0}$ in the $x y$ plane and Lorentz factor $\gamma$. The vector $\mathbf{r}=(\boldsymbol{\rho}, z)$ describes the atomic electron position; $\omega_{p}$ is the plasma frequency of the medium.

The potentials (2) satisfy the Lorenz gauge $\partial A^{\mu} / \partial x^{\mu}=0$, which sets the second line in Eq. (1) to zero. The requirement of perturbation weakness allows neglecting the term with $\mathbf{A}^{2}$ in Eq. (1) as well. Hence, the perturbation operator can be presented in the following form:

$$
\hat{\mathrm{V}}(\mathbf{r}, t)=e A^{0}\left(1-\frac{\mathrm{v}}{m c^{2}} \hat{\mathrm{p}}_{z}\right) .
$$

We assume that, before the interaction with the incident particle, the atomic electron is in the ground state with energy $\varepsilon_{1}$ and wave function $\phi_{1}(\mathbf{r}) \exp \left(-i \varepsilon_{1} t / \hbar\right)$. Further, the denomination $\phi_{k}(\mathbf{r})=|k\rangle$ will be used. The probability amplitude for this electron to transit to the $k$ th exited state as a result of interaction in the leading order of perturbation theory is [17]

$$
c_{k 1}=-\frac{i}{\hbar}\left\langle k\left|\hat{\mathrm{V}}\left(\omega_{k 1}\right)\right| 1\right\rangle,
$$

where

$$
\hat{\mathrm{V}}\left(\omega_{k 1}\right)=\int_{-\infty}^{+\infty} d t e^{i \omega_{k 1} t} \hat{\mathrm{V}}(\mathbf{r}, t)
$$


is the perturbation Fourier component and $\omega_{k 1}=$ $\left(\varepsilon_{k}-\varepsilon_{1}\right) / \hbar$.

Substituting Eqs. (2) and (3) into Eq. (4), we finally obtain

$c_{k 1}=-\frac{2 i e^{2}}{\hbar \mathrm{v}} x_{k 1}\left\{\frac{i \omega_{k 1}}{\mathrm{v} \gamma^{2}} K_{0}\left(\Omega_{k 1} \rho_{0}\right)+\Omega_{k 1} K_{1}\left(\Omega_{k 1} \rho_{0}\right)\right\}$,

where $\Omega_{k 1}^{2}=\omega_{k 1}^{2} /\left(\mathrm{v}^{2} \gamma^{2}\right)+\omega_{p}^{2} / c^{2}, K_{i}$ are the Macdonald functions, and $x_{k 1}=\langle k|x| 1\rangle$. Here, it was assumed that the incident electron moves in the $x z$ plane. In order to obtain Eq. (6), we made an expansion with respect to the small parameters $\Omega_{k 1} \rho$ and $\omega_{k 1} z / v$, which are present in the explicit expression for $\hat{\mathrm{V}}\left(\omega_{k 1}\right)$. Calculating the matrix element from the second term in Eq. (3), we applied the relation $\left\langle k\left|\hat{\mathrm{p}}_{z}\right| 1\right\rangle=m\left\langle k\left|\hat{\mathrm{v}}_{z}\right| 1\right\rangle=i m \omega_{k 1} z_{k 1}$. Finally, due to the spherical symmetry of the atom, $z_{k 1}=x_{k 1}$.

The ionization loss per unit path can be calculated as

$\frac{d \epsilon}{d z}=n \hbar \int 2 \pi \rho_{0} d \rho_{0}\left\{\sum_{k}\left|c_{k 1}\right|^{2} \omega_{k 1}+\int\left|c_{\kappa 1}\right|^{2} \omega_{\kappa 1} d^{3} \kappa\right\}$,

where the first term is associated with the atomic excitation and the second one with the ionization $(\kappa$ is the wave vector of the knocked out atomic electron). Further, to be short, we will write where possible just the first term in the braces in Eq. (7), assuming the presence of the second one by default.

It is convenient to change the order of the summation (or integration with respect to $\kappa$ ) and integration with respect to $\rho_{0}$. In order to calculate the latter integral from the second term in Eq. (6), it is convenient to proceed to the momentum representation, writing $K_{1}$ in the form

$$
K_{1}\left(\Omega_{k 1} \rho_{0}\right)=-\frac{i}{2 \pi \Omega_{k 1}} \frac{\rho_{0}}{\rho_{0}} \int d^{2} \mathrm{q} \frac{\mathbf{q} e^{i \mathbf{q} \rho_{0}}}{\mathrm{q}^{2}+\Omega_{k 1}^{2}}
$$

and presenting $\left|K_{1}\right|^{2}$ as a product of Eq. (8), with the integration variable $\mathbf{q}$ and its complex conjugate with the corresponding variable $\mathbf{q}^{\prime}$. The integrals with respect to $\boldsymbol{\rho}_{0}$ and $\mathbf{q}^{\prime}$ are easily calculated. The upper limit in the remaining integral with respect to $\mathbf{q}$ should be chosen to equal $\mathrm{q}_{0}$, reflecting the fact that one considers the restricted ionization loss. Generally, the precise value of $\mathrm{q}_{0}$ depends on the conditions of the experimental measurements. For theoretical estimations, it is usually taken equal to the inverse value of the interatomic distance $\mathrm{q}_{0} \sim 10^{8} \mathrm{~cm}^{-1}$. In this case, for $q>q_{0}$ the interaction of the incident and atomic electrons should be treated as free-particle scattering in vacuum [12]. However, for the ensemble parameters achievable at modern accelerator facilities, the coherent effects in atomic ionization take place only for soft collisions with $\mathrm{q}<\mathrm{q}_{0}$. Thus, the treatment of these effects is exhausted by the consideration of the restricted ionization loss.

The integral from the first term in Eq. (6) is calculated straightforwardly. As a result, we get

$\frac{d \epsilon}{d z}=\frac{4 \pi n e^{4}}{\hbar \mathrm{v}^{2}} \sum_{k} \omega_{k 1}\left|x_{k 1}\right|^{2}\left\{\frac{\omega_{k 1}^{2}}{\Omega_{k 1}^{2} \mathrm{v}^{2} \gamma^{4}}+2\left[\ln \frac{\mathrm{q}_{0}}{\Omega_{k 1}}-\frac{1}{2}\right]\right\}$.

For $\gamma \gg 1$, the first term in the braces here, which originates from the first term in Eq. (6), is much smaller than the second one. Further, we will neglect this term in the expressions for both $c_{k 1}$ and $d \epsilon / d z$.

The sum over $k$ in Eq. (9), which, by agreement, contains the integration over the continuous spectrum states as well, can be calculated with the use of the theorem $[12,17]$

$$
\sum_{k} \omega_{k 1}\left|x_{k 1}\right|^{2}=\hbar Z /(2 m)
$$

where $Z$ is the atomic number of the substance. With the use of Eq. (10), the mean ionization potential $I$ is defined as [12]

$$
\ln I=\frac{2 m}{\hbar Z} \sum_{k} \omega_{k 1}\left|x_{k 1}\right|^{2} \ln \omega_{k 1} .
$$

In the case of $\gamma \ll I / \omega_{p}$, expression (9) turns into

$$
\frac{d \epsilon}{d z}=\frac{4 \pi n e^{4} Z}{m \mathrm{v}^{2}}\left\{\ln \frac{\mathrm{q}_{0} \mathrm{v} \gamma}{I}-\frac{v^{2}}{2 c^{2}}\right\} .
$$

In this case of moderate $\gamma$, we kept the first term in (9). Formula (12) coincides with the well-known expression for particle ionization loss due to distant collisions [12]. Up to the terms on the order of unity in the braces, it coincides with the Bethe-Bloch formula (its part due to distant collisions) without the density effect correction.

In the opposite case of $\gamma \gg I / \omega_{p}$, formula (9) is reduced to

$$
\frac{d \epsilon}{d z}=\frac{4 \pi n e^{4} Z}{m v^{2}}\left\{\ln \frac{\mathrm{q}_{0}}{\omega_{p}}-\frac{1}{2}\right\}
$$

which is the high-energy asymptotics of the Bethe-Bloch formula with the density effect correction $[10,11]$.

\section{GENERAL EXPRESSIONS FOR THE ELECTRON ENSEMBLE IONIZATION LOSS}

After testing the discussed calculation method in the case of a single particle, let us proceed to the consideration of ionization loss by an electron ensemble consisting of a large number of particles. Let all the electrons from the ensemble move along the $z$ axis with velocity $\mathrm{v}$. The coordinates of the $r$ th electron we denote as 
$\left(\mathbf{R}+\boldsymbol{\rho}_{r}, \mathrm{v} t+z_{r}\right)$. Here, the set $(\mathbf{R}, \mathrm{v} t)$ defines the position of some point inside the ensemble which is at rest relative to the incident particles, while $\left(\boldsymbol{\rho}_{r}, z_{r}\right)$ are the electron coordinates relative to this point. For axially symmetric ensembles, it is natural to locate the point $(\mathbf{R}, \mathrm{v} t)$ at the ensemble central axis (the exact value of its $z$ coordinate does not influence the calculation results), so that $|\mathbf{R}|$ denotes the distance between this axis and the $z$ axis. With the use of Eq. (8), expression (6) for a single $r$ th electron from the ensemble can be generalized as follows:

$$
c_{k 1}^{r}=-\frac{e^{2} e^{-i \omega_{k 1} z_{r} / \mathrm{v}}}{\pi \mathrm{v} \hbar}\left(\boldsymbol{\rho} \mathbf{e}_{\rho_{0}}\right)_{k 1} \mathbf{e}_{\rho_{0}} \int d^{2} \mathrm{q} \frac{\mathbf{q} e^{i \boldsymbol{q} \rho_{0}}}{\mathrm{q}^{2}+\Omega_{k 1}^{2}},
$$

where $\mathbf{e}_{\rho_{0}}=\boldsymbol{\rho}_{0} / \rho_{0}$ and $\boldsymbol{\rho}_{0}=\mathbf{R}+\boldsymbol{\rho}_{r}$. The result of integration in Eq. (14), which we denote by the vector $\mathbf{Q}$, is directed along $\boldsymbol{\rho}_{0}$. Therefore, $\mathbf{Q} \mathbf{e}_{\rho_{0}}\left(\boldsymbol{\rho} \mathbf{e}_{\rho_{0}}\right)_{k 1}=\mathbf{Q} \boldsymbol{\rho}_{k 1}$ and the quantity $c_{k 1}$ for the whole ensemble can be written in the form in which the matrix element of the atomic electron coordinate $\rho$ is carried out from the sum over $r$ :

$c_{k 1}=-\frac{e^{2}}{\pi \mathrm{v} \hbar} \boldsymbol{\rho}_{k 1} \int d^{2} \mathrm{q} \frac{\mathbf{q} e^{i \mathbf{q R}}}{\mathrm{q}^{2}+\Omega_{k 1}^{2}} \sum_{r} e^{-i \omega_{k 1} z_{r} / \mathrm{v}+i \mathbf{q} \boldsymbol{\rho}_{\mathbf{r}}}$.

Let us assume that the particle distribution in the ensemble is axially symmetric with respect to some axis parallel to $\mathbf{v}$. In this case, the result of integration in Eq. (15) is directed along $\mathbf{R}$. Choosing this direction to be parallel to the $x$ axis and applying a procedure analogous to the one described before Eq. (9), one can finally present the expression for the ensemble ionization loss in the following form:

$$
\begin{aligned}
\frac{d \epsilon}{d z}= & \frac{4 n e^{4}}{\mathrm{v}^{2} \hbar} \sum_{k} \omega_{k 1}\left|x_{k 1}\right|^{2} \int d^{2} \mathrm{q} \frac{\mathrm{q}^{2}}{\left(\mathrm{q}^{2}+\Omega_{k 1}^{2}\right)^{2}} \\
& \times \sum_{r, p} e^{-i \omega_{k 1}\left(z_{r}-z_{p}\right) / \mathrm{v}+i \mathbf{q}\left(\boldsymbol{\rho}_{r}-\boldsymbol{\rho}_{p}\right)} .
\end{aligned}
$$

Note that, generally, for atoms with $Z \geq 2$ it is necessary to perform here a summation over all the atomic electrons as well. Further, for the sake of simplicity, we will still write the expressions for the case of a single electron in the atom. Proceeding to the approximation of a continuous charge distribution in the ensemble and, where possible, calculating the integral with respect to $\mathbf{q}$, we get

$$
\frac{d \epsilon}{d z}=\frac{4 \pi n Z e^{4}}{m v^{2}} N\left\{\ln \frac{\mathrm{q}_{0}}{\bar{\Omega}}-\frac{1}{2}+(N-1) G\left(\gamma, \omega_{p}\right)\right\},
$$

where $N$ is the total number of particles in the ensemble and the quantity $\bar{\Omega}$ is defined by analogy with the mean ionization potential:

$$
\ln \bar{\Omega}=\frac{2 m}{\hbar Z} \sum_{k} \omega_{k 1}\left|x_{k 1}\right|^{2} \ln \Omega_{k 1}
$$

The function $G\left(\gamma, \omega_{p}\right)$, which also depends on the structure of the excited levels of the atom and the ensemble geometrical properties, has the form

$G\left(\gamma, \omega_{p}\right)=\frac{2 m}{Z \hbar} \sum_{k} \omega_{k 1}\left|x_{k 1}\right|^{2} F_{\|}\left(\omega_{k 1}\right) \int d \mathrm{q} \frac{\mathrm{q}^{3} F_{\perp}(q)}{\left(\mathrm{q}^{2}+\Omega_{k 1}^{2}\right)^{2}}$,

where $F_{\|}$and $F_{\perp}$ are the ensemble form factors, which are defined as

$$
\begin{aligned}
& F_{\|}\left(\omega_{k 1}\right)=\left|\int_{-\infty}^{+\infty} d z f_{\|}(z) e^{-i \omega_{k 1} z / v}\right|^{2}, \\
& F_{\perp}(q)=\left|2 \pi \int_{0}^{\infty} d r r J_{0}(\mathrm{q} r) f_{\perp}(r)\right|^{2},
\end{aligned}
$$

respectively, and $J_{0}$ is the Bessel function. The functions $f_{\|}$ and $f_{\perp}$ describe, respectively, the longitudinal and transversal particle distribution in the ensemble (we assume that the particle distribution factorizes in these directions) and are normalized to unity.

The first two terms in braces in Eq. (17) are associated with the incoherent part of the ensemble ionization loss. Being proportional to $N$, it is just the sum of the independent losses by separate particles defined by the Bethe-Bloch formula. The part of the expression (17) which contains $G\left(\gamma, \omega_{p}\right)$ is the coherent part of the loss. It is proportional to $N^{2}$ and describes the influence of the interference (coherent) effects upon the ensemble ionization loss. According to Ref. [6] and more rigorous calculations in the present work, these effects can enhance the value of the ensemble ionization loss by several orders of magnitude (for currently available parameters of the electron ensembles) compared to the result predicted by the Bethe-Bloch formula.

The expression $2 m \omega_{k 1}\left|x_{k 1}\right|^{2} /(Z \hbar)$, the sum of which over $k$ is equal to unity, can be considered as a distribution function over the excited states of the atomic electron (which includes both the states of the discrete and continuous spectrum). Thus, Eq. (19) represents the averaging of the function depending on $F_{\|}$and $F_{\perp}$ over the atomic electron states. Further, we will denote such an averaging by $\langle\ldots\rangle$. Under the condition of the full value density effect $\left(\gamma \gg I / \omega_{p}\right)$, the expression for $G\left(\gamma, \omega_{p}\right)$ simplifies. Namely, in this case, $\Omega_{k 1}=\omega_{p} / c$ and $G$ ceases to depend on $\gamma$, while the averaging involves just the longitudinal form factor: 


$$
G\left(\omega_{p}\right)=\left\langle F_{\|}\left(\omega_{k 1}\right)\right\rangle \int d \mathrm{q} \frac{\mathrm{q}^{3} F_{\perp}(q)}{\left(\mathrm{q}^{2}+\omega_{p}^{2} / c^{2}\right)^{2}} .
$$

Further, we will mainly concentrate on this particular case.

\section{APPLICABILITY CONDITIONS FOR THE APPLIED APPROACH}

In the present work, we study the case when, due to coherent effects, the interaction of incident particles with atomic electrons can be much more intense than in the case of a single electron scattering on an atom. Therefore, it is necessary to study the applicability of perturbation theory which is applied in the present investigation.

The general condition of the workability of the applied approach is $\sum_{k=2}^{\infty}\left|c_{k 1}\right|^{2} \ll 1$. The condition of the convergence of the sum over $k$ suggests that by an order of magnitude this sum coincides with the sum of the first several terms in it. In the present work, numerical estimations will be made for hydrogen atoms. In this case, due to the quick decrease of $\left|x_{k 1}\right|^{2}$ with the increase of $k$ (see details below), the above condition with nice accuracy can be replaced by

$$
\left|c_{21}\right|^{2} \ll 1
$$

which includes just the probability of the electron transition to the first $(k=2)$ excited level. An exception may be made for ensembles whose longitudinal form factors $F_{\|}$turn to zero at certain finite values of the ensemble parameters, as, for instance, in the case of a homogeneous longitudinal particle distribution, briefly discussed in Sec. VA. Presently, for such values of the parameters, $\left|c_{21}\right|^{2}$ may turn to zero, while the terms with $k \geq 3$ remain nonzero. In this case, it is necessary to take into account the first two terms from the sum over $k$ in Eq. (23).

In the case of a single incident particle with unit charge, the value of $\left|c_{21}\right|^{2}$, which can be obtained from Eq. (6), is

$$
\left|c_{21}\right|^{2}=4 \alpha^{2}\left|x_{21}\right|^{2} / \rho_{0}^{2},
$$

where $\alpha=1 / 137$ is the fine-structure constant. For a hydrogen atom, the quantity $\left|x_{21}\right|^{2}$ is on the order of $a_{0}^{2}$ (more precisely, $\left.0.56 a_{0}^{2}\right)$, where $a_{0}=\hbar^{2} /\left(m e^{2}\right)$ is the Bohr radius. The minimal impact parameters $\rho_{0}$, associated with the maximum values of $\left|c_{21}\right|^{2}$, which we consider in our study are $\rho_{0} \sim a_{0}$. This gives $\left|c_{21}\right|^{2} \sim \alpha^{2}$, providing nice validation of the condition (23).

Let us now calculate the quantity $\left|c_{k 1}\right|^{2}$ (for an arbitrary value of $k$ ) for the case of an incident ensemble on the basis of Eq. (15). Presently, we need to obtain the result for an arbitrary value of $\mathrm{R}$ (which is the distance between the atom and the ensemble central axis) without preliminary integration with respect to $\mathbf{R}$. In the previous section, such an integration (in that case, it was performed with respect to $\boldsymbol{\rho}_{0}$ ) preceded the ones with respect to $\mathbf{q}$ and $\mathbf{q}^{\prime}$ and considerably simplified the calculation of $d \epsilon / d z$. Proceeding from the sum over the discrete set of charges to the approximation of a continuous charge distribution, as was done in the case of $d \epsilon / d z$ calculation, one can present Eq. (15) in the following form:

$$
\left|c_{k 1}\right|^{2}=\left(\frac{\alpha}{\pi}\right)^{2}\left|x_{k 1}\right|^{2} N^{2} F_{\|}\left(\omega_{k 1}\right)\left|\mathbf{I}_{k 1}(\mathbf{R})\right|^{2},
$$

where

$$
\mathbf{I}_{k 1}(\mathbf{R})=\int d^{2} \mathbf{q} \frac{\mathbf{q} e^{i \mathbf{q} \mathbf{R}}}{\mathbf{q}^{2}+\Omega_{k 1}^{2}} \int d^{2} r f_{\perp}(r) e^{i \mathbf{q r}}
$$

Here, the incoherent part of $\left|c_{k 1}\right|^{2}$, which is proportional to $N$, is neglected compared to the coherent one proportional to $N^{2}$. This is due to the fact that we are mainly interested in the study of the applicability condition (23) in the case when the coherent effects in the ensemble ionization loss are rather huge. The integral $\mathbf{I}_{k 1}(\mathbf{R})$ is directed along $\mathbf{R}$, which allows one to present it as

$\mathbf{I}_{k 1}(\mathbf{R})=(2 \pi)^{2} i \mathbf{e}_{R} \int_{0}^{\infty} d r r f_{\perp}(r) \int_{0}^{\infty} d \mathrm{q} \frac{\mathrm{q}^{2} J_{0}(\mathrm{q} r) J_{1}(\mathrm{qR})}{\mathrm{q}^{2}+\Omega_{k 1}^{2}}$,

where $\mathbf{e}_{R}=\mathbf{R} / \mathrm{R}$. After calculation of the integral with respect to q in Eq. (27), expression (25) acquires the form

$$
\left|c_{k 1}\right|^{2}=16 \pi^{2} \alpha^{2}\left|x_{k 1}\right|^{2} N^{2} \Omega_{k 1}^{2} F_{\|}\left(\omega_{k 1}\right)\left|S_{k 1}(\mathrm{R})\right|^{2},
$$

with

$$
\begin{aligned}
S_{k 1}(\mathrm{R})= & K_{1}\left(\Omega_{k 1} \mathrm{R}\right) \int_{0}^{R} d r r f_{\perp}(r) I_{0}\left(\Omega_{k 1} r\right) \\
& -I_{1}\left(\Omega_{k 1} \mathrm{R}\right) \int_{R}^{\infty} d r r f_{\perp}(r) K_{0}\left(\Omega_{k 1} r\right) .
\end{aligned}
$$

Here, $I_{i}$ are the Bessel functions of an imaginary argument (like the Macdonald functions $K_{i}$ ).

The case of a single incident particle can be obtained from Eq. (28) by putting $f_{\perp}(r)=\delta(\mathbf{r}), N=1$, and $F_{\|}\left(\omega_{k 1}\right)=1$. In this case, for $R \ll \Omega_{k 1}^{-1}$ and $k=2$, expression (28) reduces to Eq. (24) with $\rho_{0}=\mathrm{R}$.

Let us, for the sake of illustration, calculate $\left|c_{21}\right|^{2}$ for the simplest case of an ensemble (bunch) with homogeneous transversal particle distribution. Presently, $f_{\perp}(r)=$ $\theta(d-r) /\left(\pi d^{2}\right)$, where $\theta(x)$ is the Heaviside step function and $d$ is the bunch radius. We will put $\mathrm{R}=d$, since in this case $\left|c_{k 1}\right|^{2}$ reaches its maximum value. From Eqs. (28) and (29), we have 
$\left|c_{k 1}\right|^{2}=16 \alpha^{2} \frac{\left|x_{k 1}\right|^{2}}{d^{2}} N^{2} F_{\|}\left(\omega_{k 1}\right) K_{1}^{2}\left(\Omega_{k 1} d\right) I_{1}^{2}\left(\Omega_{k 1} d\right)$.

Further, we will be interested, particularly, in the case when the full value density effect $\left(\gamma \gg I / \omega_{p}\right)$ takes place in a hydrogen medium and the typical bunch radius is $d \sim c / \omega_{p}$. In this case, Eq. (30) reads $(k=2)$

$$
\left|c_{21}\right|^{2} \sim \alpha^{2} N^{2}\left(a_{0} \omega_{p} / c\right)^{2} F_{\|}\left(\omega_{21}\right) .
$$

Here, we see that for the electron ensemble $\left|c_{21}\right|^{2}$ is not just $\alpha^{2} N^{2}$, as would be, e.g., for a heavy ion with charge $N e$. Expression (31) also contains two "suppression" factors which considerably reduce its value. Indeed, for hydrogen under normal conditions the ratio $a_{0} \omega_{p} / c$ is about $5 \times 10^{-5}$. For bunches with length noticeably exceeding the value $c / \omega_{21}$, the longitudinal form factor $F_{\|}$is much less than unity as well (though the coherent effects can still be considerable in this case). Therefore, we see that the applied approach, based on perturbation theory, can be applicable even for very large values of $N$ typical for macroscopic particle ensembles.

\section{NUMERICAL ESTIMATION OF THE IONIZATION LOSS BY ELECTRON ENSEMBLES IN HYDROGEN}

In this section, we perform a numerical calculation of restricted ionization loss by ultrarelativistic electron ensembles (bunches). The parameters of the ensembles are chosen to correspond to the ones which are available at modern accelerator facilities. The cases of various shapes of the bunches are considered. As noted, the calculations are made for the simplest case of the substance, which corresponds to atomic hydrogen, for which the precise consideration is possible.

The major quantities in (17)-(21) describing the substance, which the ensemble penetrates, are $\omega_{k 1}$ and $x_{k 1}$. For the discrete spectrum of atomic hydrogen, the quantities $\omega_{k 1}$ are

$$
\omega_{k 1}=\frac{m e^{4}}{2 \hbar^{3}} \frac{k^{2}-1}{k^{2}},
$$

which follows from the well-known formula for $\varepsilon_{k}$. Hence, for transitions to the continuous spectrum,

$$
\omega_{\kappa 1}=\frac{m e^{4}}{2 \hbar^{3}}+\frac{\hbar \kappa^{2}}{2 m} .
$$

The matrix elements $x_{k 1}$ for the discrete spectrum can be directly obtained, e.g., from the matrix elements $\left(e^{i \mathbf{q r}}\right)_{k 1}$, derived in Ref. [17]. They read (only transitions to states with $p=0$, described by wave functions in parabolic coordinates $\phi_{n_{1}, n_{2}, p}$, take place; $n_{1}+n_{2}=k-1$ )

$$
\left|x_{k 1}\right|^{2}=2^{8} k^{7} a_{0}^{2} \frac{(k-1)^{2 k-6}}{(k+1)^{2 k+6}} \frac{k^{2}-1}{3} .
$$

The matrix elements $x_{\kappa 1}$ can be derived with the use of wave functions of the continuous spectrum in parabolic coordinates [17]. They read

$\left|x_{\kappa 1}\right|^{2}=\frac{2^{8} a_{0}^{4}}{3 \kappa} \frac{\exp \left[-\frac{4}{\kappa a_{0}} \operatorname{arctg}\left(\kappa a_{0}\right)\right]}{\left(1+\kappa^{2} a_{0}^{2}\right)^{5}\left\{1-\exp \left[-2 \pi /\left(\kappa a_{0}\right)\right]\right\}}$.

The numerical estimation of the sum $\sum \omega_{k 1}\left|x_{k 1}\right|^{2}$ with the use of (32)-(35), including integration over the continuous spectrum states, is in agreement with Eq. (10).

\section{A. Ensemble with a Gaussian particle distribution}

Among other parameters, the magnitude of electron ensemble $d \epsilon / d z$ is defined by its form factors. Thus, as in the case of radiation, the magnitude of the coherent effects in the ionization loss significantly depends on the spatial distribution of the electrons in the ensemble. In the present subsection, we perform a numerical estimation of the ensemble ionization loss for the case of Gaussian particle distribution in both the longitudinal (along the $z$ axis) and transversal directions. Presently, the functions $f_{\|}$ and $f_{\perp}$ can be written, respectively, as [18]

$$
f_{\|}(z)=e^{-z^{2} / 2 l^{2}} / \sqrt{2 \pi} l, \quad f_{\perp}(r)=e^{-r^{2} / 2 d^{2}} / 2 \pi d^{2}
$$

and result in the following forms of $F_{\|}$and $F_{\perp}$ :

$$
F_{\|}\left(\omega_{k 1}\right)=e^{-\omega_{k 1}^{2} l^{2} / \mathrm{v}^{2}}, \quad F_{\perp}(\mathrm{q})=e^{-\mathrm{q}^{2} d^{2}} .
$$

The quantities $l$ and $d$ describe the ensemble dimensions in the longitudinal and transversal directions, respectively. They are related to the corresponding full widths at half maxima (FWHM) $l_{f}$ and $d_{f}$ as $l_{f}=l \sqrt{8 \ln 2}$ and $d_{f}=d \sqrt{8 \ln 2}$.

Let us consider the case of fairly high-energy incident electrons $\left(\gamma \gg I / \omega_{p}\right)$ when the full value density effect takes place. Calculating the integral with respect to $\mathrm{q}$ in Eq. (22), we finally obtain

$\frac{d \epsilon}{d z}=\frac{4 \pi n e^{4}}{m \mathrm{v}^{2}} N\left\{\ln \frac{c \mathrm{q}_{0}}{\omega_{p}}-\frac{1}{2}-\frac{N}{2} g\left(\omega_{p}, d\right)\left\langle e^{-\omega_{k 1}^{2} l^{2} / \mathrm{v}^{2}}\right\rangle\right\}$,

where

$g\left(\omega_{p}, d\right)=e^{\omega_{p}^{2} d^{2} / c^{2}}\left(1+\omega_{p}^{2} d^{2} / c^{2}\right) \operatorname{Ei}\left(-\omega_{p}^{2} d^{2} / c^{2}\right)+1$

and $\mathrm{Ei}$ is the integral exponent function. The function $-g\left(\omega_{p}, d\right)$ monotonically decreases with the increase of $d$. Namely, for $x \gg 1$ we have $\operatorname{Ei}(-x) \approx e^{-x}\left(-x^{-1}+x^{-2}-\right.$ $\left.2 x^{-3}\right)$, which leads to $-g\left(\omega_{p}, d\right) \approx\left(c / \omega_{p} d\right)^{4}$ for 
$d \gg c / \omega_{p}$. Note that, in order to obtain the expression for $d \epsilon / d z$ in the general case of arbitrary $\gamma$ (provided $\gamma \gg 1$ ), it is necessary to make the substitution $\omega_{p} / c \rightarrow \Omega_{k 1}$ in Eq. (39) and move function $g$ under angle brackets. The substitution $\omega_{p} / c \rightarrow \bar{\Omega}$ in the first term in Eq. (38) is required in this case as well.

By analogy with the mean ionization potential (11), it is presently possible to introduce some effective mean ionization potential $\bar{\omega}$ defined as

$$
e^{-\bar{\omega}^{2} l^{2} / \mathrm{v}^{2}}=\left\langle e^{-\omega_{k 1}^{2} l^{2} / \mathrm{v}^{2}}\right\rangle
$$

or, explicitly,

$$
\bar{\omega}=\frac{\mathrm{v}}{l} \sqrt{-\ln \left\{\frac{2 m}{\hbar} \sum_{k} \omega_{k 1}\left|x_{k 1}\right|^{2} e^{-\omega_{k 1}^{2} l^{2} / \mathrm{v}^{2}}\right\}} .
$$

If we substitute (40) in (38), the latter expression will acquire the same form as the analogous expression for Gaussian bunch ionization loss in an ultrathin solid target obtained in Ref. [6] with the use of a simplified classical approach. The main difference between these expressions, which emphasizes the main advantage of our present consideration, concerns the quantity $\bar{\omega}$. Namely, in Ref. [6], for each atomic shell $\bar{\omega}$ was chosen equal to the mean ionization potential of this shell (e.g., for silicon targets, such potentials for K, L, and M shells were taken from Ref. [19]). The present consideration provides a principal possibility to calculate these quantities on the basis of Eq. (41), at least approximately for most of the substances (generally, $Z \neq 1$ should be reestablished in this formula). It is a rather important advance, since, as further shown (see Fig. 3), ensemble ionization loss can be very sensitive to the value of $\bar{\omega}$. It is worth noting that formula (41) indicates that $\bar{\omega}$ is not just a constant defined by the physical properties of the medium but depends on the longitudinal dimension $l$ of the ensemble and the explicit functional dependence of its form factor $F_{\|}$. In the absence of the density effect (or under the condition of its partial manifestation at not very large $\gamma$ ), the quantity $\bar{\omega}$ is defined by the ensemble dimensions in both transversal and longitudinal directions and the corresponding form factors $F_{\|}$and $F_{\perp}$.

Figure 1 shows the dependence of $\bar{\omega}$ on the ensemble length $l_{f}$ (FWHM in the longitudinal direction) calculated on the basis of Eq. (41), where we put $\mathrm{v}=c$. The calculation is performed for atomic hydrogen with the use of Eqs. (32)-(35). The figure demonstrates a noticeable change of $\bar{\omega}$ in the region of $l_{f}$ which corresponds to the most considerable manifestation of coherent effects in ionization loss. Such a region can be defined by the condition $l<l_{0} \sim \mathrm{v} / \omega_{21}$. At $l \sim l_{0}$, according to Eq. (37), the form factor $F_{\|}$corresponding to the smallest excitation energy $\hbar \omega_{21} \approx 10.2 \mathrm{eV}$ becomes close to unity.



FIG. 1. Dependence of $\bar{\omega}$ on the ensemble longitudinal dimension $l_{f}$ in hydrogen (here, to be short, $\hbar=1$ ).

At smaller $l$, the same occurs for the rest of $\omega_{k 1}$. Presently, $l_{0} \sim 20 \mathrm{~nm}$, which corresponds to $l_{f} \sim 46 \mathrm{~nm}$.

At $l \gg l_{0}$, the quantity $\bar{\omega}$ tends to the smallest excitation energy $\omega_{21}$. The contributions from transitions to higher levels of the discrete and continuous spectrum are suppressed by the exponential factor in Eq. (41) in this case. In the opposite extreme case of small $l$, it is possible to expand the exponents in Eq. (41) and obtain the following relation: $\bar{\omega}=\left\langle\omega_{k 1}^{2}\right\rangle^{1 / 2}$. It is valid under condition $l \ll v / \omega_{\max }$, where $\omega_{\max }$ is the value of $\omega_{\kappa 1}$ at some maximum $\kappa$ which still contributes to the integral with respect to the continuous spectrum states in Eq. (41), or, more generally, in Eq. (10). For the considered case of hydrogen atoms, it is roughly $\kappa \lesssim 4 / a_{0}$ which make the main contribution to this integral. Thus, presently, $\hbar \omega_{\max }$ can be set equal to $\hbar \omega_{\kappa 1}$ with the value $\kappa=4 / a_{0}$ of the knocked out electron wave vector in Eq. (33) and is around $230 \mathrm{eV}$.

Before calculating $d \epsilon / d z$, it is necessary to define the applicability range of the applied approach under considered conditions. Presently, we will make estimations for the parameters which can be realized at the Short Innovative Bunches and Accelerators at DESY (SINBAD) facility [20], presently under construction at Deutsches ElektronenSynchrotron (DESY). This particularly includes the electron energy of $100 \mathrm{MeV}, d_{f}=0.75 \mu \mathrm{m}$ beam spot size, and $1 \mathrm{pC}$ total charge of a subfemtosecond bunch $\left(l_{f}<300 \mathrm{~nm}\right)$. Figure 2 demonstrates $\left|c_{21}\right|^{2}$ as a function of $l_{f}$ in this case calculated on the basis of Eqs. (28) and (29). We take $\hbar \omega_{p} \approx 0.19 \mathrm{eV}$, which is the plasma frequency of atomic hydrogen at normal conditions (it is $\sqrt{2}$ times smaller than the corresponding frequency for molecular hydrogen). The calculation is made for $R=0.5 \mu \mathrm{m}$, at which $\left|c_{21}\right|^{2}$, as a function of $R$, reaches its maximum. Note that we consider the case when the particle density $n_{0}$ in the center of the bunch is fixed. It corresponds to a total charge of $1 \mathrm{pC}$ at length $l_{f} \approx 130 \mathrm{~nm}(0.43 \mathrm{fs})$, which is typical for the discussed facility. The change of the bunch length occurs due to the change of the number of particles $N$. 


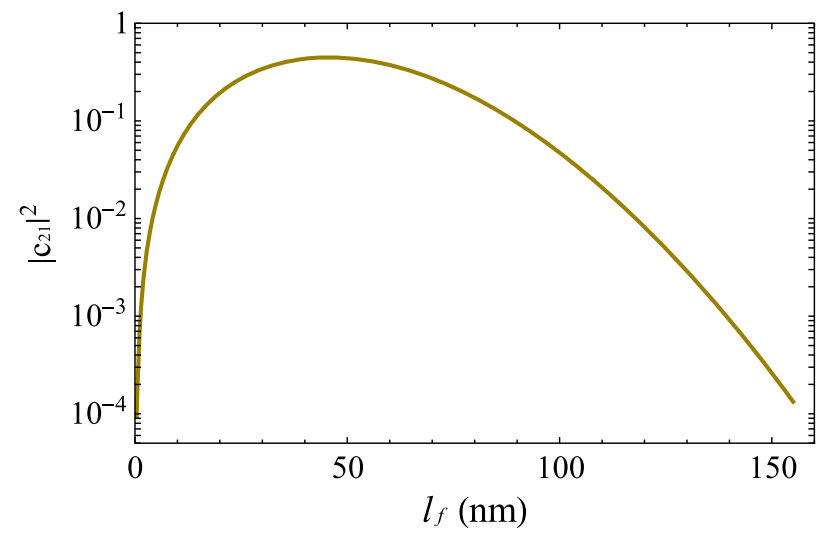

FIG. 2. Dependence of $\left|c_{21}\right|^{2}$ on $l_{f}$ for an ensemble with Gaussian particle distribution in hydrogen for $d_{f}=0.75 \mu \mathrm{m}$, $n_{0}=6 \times 10^{19} \mathrm{~cm}^{-3}$, and $\gamma \gg I / \omega_{p}$.

Here, we see that in the considered case condition (23) is satisfied rather well in the regions $l_{f} \gtrsim 80 \mathrm{~nm}$ and $l_{f} \lesssim 15 \mathrm{~nm}$. The present approach is applicable here for the calculation of ensemble ionization loss.

The dependence of ensemble ionization loss per unit particle on $l_{f}$ in the region of $l_{f} \gtrsim 80 \mathrm{~nm}$ is presented in Fig. 3 (thick green line). The dimensionless quantity on the vertical axis is defined as $d E / d z=m \mathrm{v}^{2} /\left(4 \pi n e^{4}\right) d \epsilon / d z$. The value of $\mathrm{q}_{0}$ in Eq. (38) is usually determined by experimental conditions. For estimations, it is natural to apply the value $\mathrm{q}_{0} \sim 10^{8} \mathrm{~cm}^{-1}$ [10]. In this case, the minimal considered distance between the incident particle and the atom $\rho_{0} \sim 1 / \mathrm{q}_{0}$ is on the order of $a_{0}$. The dashed line in the figure shows the ionization loss value in the absence of coherent effects, defined by the first two terms in Eq. (38) corresponding to the Bethe-Bloch result. Here, we see that for $l_{f}<170 \mathrm{~nm}$ coherent effects are manifested in ionization loss for the considered type of particle distribution in the ensemble. With the decrease of $l_{f}$, these

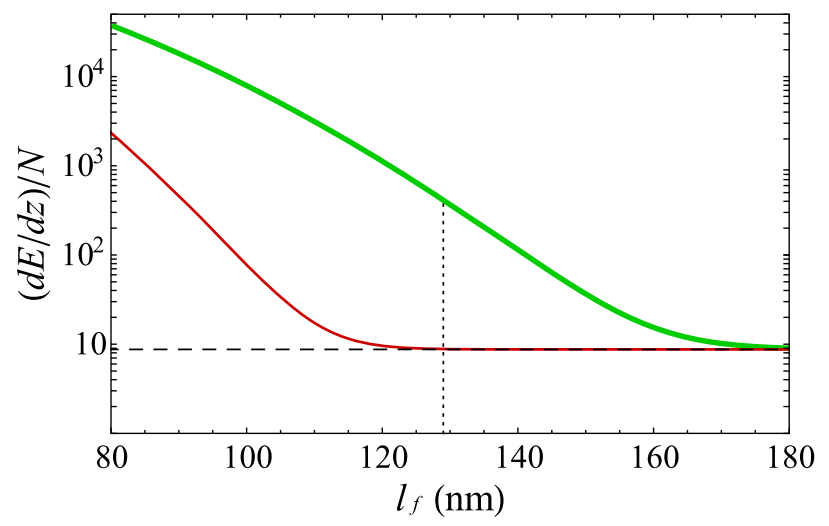

FIG. 3. Dependence of the ensemble ionization loss on $l_{f}$ in hydrogen for $d_{f}=0.75 \mu \mathrm{m}, n_{0}=6 \times 10^{19} \mathrm{~cm}^{-3}$ and Gaussian particle distribution (thick green line). Thin red line, calculation for $\bar{\omega}=I$; dashed line, no coherent effects; the dotted line marks the length $l_{f} \approx 130 \mathrm{~nm}(0.43 \mathrm{fs})$. effects become considerable and result in a dramatic increase of the $d \epsilon / d z$ value by several orders of magnitude. For the bunch length typical for the SINBAD facility, marked by the vertical line in the figure, the coherent enhancement factor of the ionization loss is still very large (about 47). By this factor, we mean the ratio of $d \epsilon / d z$ with and without taking into account coherent effects.

For comparison, we also present the result of calculation on the basis of Eq. (38) with $\bar{\omega}$ being equal to the mean ionization potential $I \approx 15 \mathrm{eV}$ (thin red line). As noted, such a result corresponds to the simplified approach applied in Ref. [6]. We see that in the considered range of $l_{f}$, where the explicit form of $F_{\|}$is decisive, the difference between the predictions of the simplified and more accurate approaches in the present case is considerable. The results of these approaches coincide in the region $l_{f} \lesssim l_{\max }=$ $\sqrt{8 \ln 2} \mathrm{v} / I$. Here, $d \epsilon / d z$ reaches its maximum value at $l_{f} \sim$ $l_{\max } \approx 30 \mathrm{~nm}$ (strictly speaking, the neighborhood of this maximum is not accurately described by the present approach, according to Fig. 2). With a further decrease of $l_{f}$, the coherent part of $d \epsilon / d z$ quickly vanishes. It is caused by the decrease of the number of particles in the ensemble (at constant $n_{0}$ ) with the decrease of $l_{f}$.

Figure 3 indicates a fast decrease of the coherent part of the ionization loss with the increase of $l_{f}$ at $l_{f}>l_{\max }$. Such a decrease can be much slower if the longitudinal particle distribution $f_{\|}$in the ensemble differs from the Gaussian one. Consider, for instance, the simplest case of this kind, which corresponds to homogeneous distribution $f_{\|}=$const. The corresponding transversal distribution $f_{\perp}$ we will still assume to be Gaussian. In this case, the calculation of $d \epsilon / d z$ can be performed with the use of Eq. (38), in which the longitudinal form factor is substituted by $F_{\|}=4 \mathrm{v}^{2} \sin ^{2}\left(\omega_{k 1} l / 2 \mathrm{v}\right) / \omega_{k 1}^{2} l^{2}$, where $l$ is the bunch length. Particularly, the coherent enhancement factor at $l \approx 130 \mathrm{~nm}$ is presently about 4800 , which is 100 times larger than in the case of the Gaussian $f_{\|}$. The value of $\left|c_{21}\right|^{2}+\left|c_{31}\right|^{2}$ for this $l$ is presently around 0.084 , which validates the application of perturbation theory in this case as well. The necessity of taking into account both $c_{21}$ and $c_{31}$ in the applicability condition in the present case has been discussed above after Eq. (23).

Let us finally note that in the considered case the applied assumption of the presence of the full value density effect (strictly valid at $\gamma \gg I / \omega_{p}$ ) is somewhat approximate, since $I / \omega_{p} \approx 80$, while $\gamma \approx 200$. Nevertheless, a more accurate calculation on the basis of Eqs. (17) and (19) leads to the result, which is almost undistinguishable from the one presented in Fig. 3.

\section{B. Ensemble with a periodically modulated particle density. Resonance effect}

X-ray and ultraviolet free-electron lasers (FELs) are facilities which allow obtaining high-energy electron 
ensembles of an extremely small size. This is achieved via the phenomenon of microbunching of the initial incident electron bunch when it moves in the undulator section of a FEL. In this case, the initial bunch breaks up into a train of short microbunches, whose length is equal to the emitted coherent radiation wavelength. In Ref. [6], it was shown (with the use of a simplified classical approach) that in the case where such an ensemble interacts with matter, generally, the ionization losses of its separate microbunches may not be independent. Interference between the contributions of these microbunches to the total energy transmitted to an atomic electron may result in the resonant amplification of this energy for certain values of the microbunching period. In the present subsection, we study this effect with the use of the more accurate approach developed in the present work and make numerical estimations for the case of a hydrogen medium.

As a model of a microbunched incident beam, we consider an electron ensemble with the density periodically modulated in the longitudinal direction $f_{\|}=$ $[1-\cos (2 \pi z / l)] /(s l)$. It is, certainly, a somewhat idealized model of the real electron density which can be achieved at a FEL. Presently, $l$ is the microbunching period, and $s$ is the total number of microbunches in the ensemble. By $L=s l$ we denote the total length of the ensemble. In this case, the longitudinal form factor reads

$F_{\|}\left(\omega_{k 1}\right)=\left(\frac{2 \mathrm{v}}{\omega_{k 1} L}\right)^{2}\left(\frac{4 \pi^{2} / l^{2}}{\omega_{k 1}^{2} / \mathrm{v}^{2}-4 \pi^{2} / l^{2}}\right)^{2} \sin ^{2}\left(\frac{\omega_{k 1} L}{2 \mathrm{v}}\right)$.

We still assume that transversal particle distribution $f_{\perp}$ is Gaussian and the full value density effect takes place. The ensemble ionization loss in this case can be calculated with the use of Eq. (38), in which the longitudinal form factor is substituted by Eq. (42). As before, the expression $\left\langle F_{\|}\right\rangle$ consists of the sum over the states of the discrete spectrum and integration over the states of the continuous one: $\left\langle F_{\|}\right\rangle=\left\langle F_{\|}\right\rangle_{\text {discr }}+\left\langle F_{\|}\right\rangle_{\text {cont }}$. If $f_{\|}$is periodically modulated, the function $F_{\|}$possesses sharp maxima, as, e.g., at $\omega_{k 1}=$ $2 \pi \mathrm{v} / l$ in Eq. (42). In this case, the integral with respect to $\kappa$ in $\left\langle F_{\|}\right\rangle_{\text {cont }}$ can be analytically calculated. Indeed, with the use of Eqs. (33) and (35), it can be explicitly written in the form

$$
\left\langle F_{\|}\right\rangle_{\mathrm{cont}}=\frac{2^{8}}{3} \int_{0}^{\infty} d x x R(x) F_{\|}\left(\omega_{\kappa 1}\right),
$$

where $x=\kappa a_{0}, \omega_{\kappa 1}=m e^{4}\left(x^{2}+1\right) / 2 \hbar^{3}$, and

$$
R(x)=\frac{\exp [-4 \operatorname{arctg} x / x]}{\left(1+x^{2}\right)^{4}(1-\exp [-2 \pi / x])} .
$$

For a fixed value of $l$, the main contribution to the integral in Eq. (43) is made by the narrow region around the point $x=x_{0}=\left[4 \pi \mathrm{v} \hbar^{3} /\left(m e^{4} l\right)-1\right]^{1 / 2}$, which corresponds to $\omega_{\kappa 1}=2 \pi \mathrm{v} / l$. This allows one to extract all the factors from the integral except the sine squared and the denominator $\left(\omega_{k 1} / \mathrm{v}-2 \pi / l\right)^{2}$, which are the constituent parts of $F_{\|}$. After calculation of the remaining integral, Eq. (43) acquires the form

$$
\left\langle F_{\|}\right\rangle_{\mathrm{cont}}=\frac{2^{7} \pi \hbar^{3} \mathrm{v}}{3 m e^{4} L} R\left(x_{0}\right) .
$$

Note that this expression is valid if $l<l_{b}=4 \pi \mathrm{v} \hbar^{3} /\left(m e^{4}\right)$. For $\mathrm{v} \approx c$, we have $l_{b} \approx 91 \mathrm{~nm}$. At $l>l_{b}$, the contribution of $\left\langle F_{\|}\right\rangle_{\text {cont }}$ to $\left\langle F_{\|}\right\rangle$is negligibly small. The value $l_{b}$ marks the boundary between the regions of $l$ in which resonance effects take place for atomic excitation (transitions to the discrete spectrum states) and ionization (transitions to the continuous spectrum).

Numerical estimations of the microbunched ensemble ionization loss will be made for the parameters, which can be realized at the European $\mathrm{x}$-ray FEL $[21,22]$. This includes a multi-GeV electron energy (the condition $\gamma \gg$ $I / \omega_{p}$ is nicely satisfied in this case, and the precise value of the energy is not significant), the bunch length of $L=24 \mu \mathrm{m}$, and its total charge of $1 \mathrm{nC}$. However, we will consider a much wider range of microbunching periods $l$ (corresponding rather to the operational range of ultraviolet FELs) than the one typical for this FEL. This is due to the fact that for hydrogen atoms, which we consider as an example in our present study, the resonance effects are most significant for $l=2 \pi \mathrm{v} / \omega_{k 1}$, corresponding to the ultraviolet range of the excitation and ionization frequencies $\omega_{k 1}$. Such effects "expand" to the soft x-ray region for heavier substances with larger $Z$.

Figure 4 demonstrates the dependence of ensemble ionization loss on the microbunching period $l$. Note that for a fixed $L$ the quantity $l$ changes discretely, since the

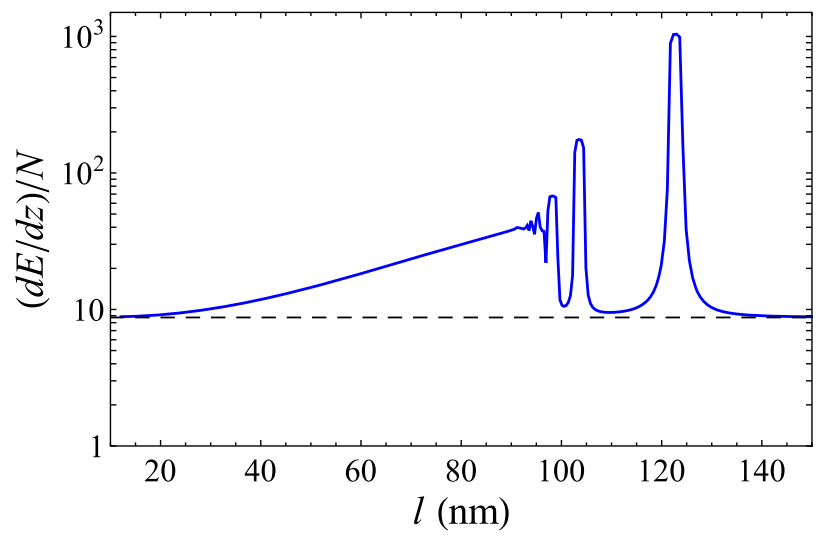

FIG. 4. Dependence of the ensemble ionization loss on the microbunching period $l$ in hydrogen for $d_{f}=40 \mu \mathrm{m}$, $L=24 \mu \mathrm{m}$, and $\gamma \gg I / \omega_{p}$ (solid line). Dashed line, no coherent effects. 
number of microbunches $s$ is an integer, and the presented dependence is extrapolated to the continuous set of $l$. Moreover, the figure shows the result which is averaged with respect to a small variation $\delta L$ of the length $L$. Presently, $\delta L / L=0.02$. We see that in the region $l>l_{b}$ there exists a series of resonances associated with the frequencies $\omega_{k 1}$ of the atomic electron transition to the excited states of the discrete spectrum. The values of $d \epsilon / d z$ in these resonances considerably exceed the ensemble ionization loss without taking into account the coherent effects. The value of $F_{\|}$in each resonance is $1 / 4$. Thus, the ratio of $d \epsilon / d z$ values in the resonances is defined by the ratio of the corresponding $\omega_{k 1}\left|x_{k 1}\right|^{2}$ values. However, this statement is somewhat approximate due to some blurring of the peaks as a result of averaging with respect to $\delta L$. Some additional blurring may also occur due to the natural width of the excited atomic levels, which we do not presently take into account. Note that the maximum value of $\left|c_{21}\right|^{2}$, which is reached at the position of the rightmost resonance $(l \approx 122 \mathrm{~nm})$, is about 0.054 and perturbation theory is well applicable in the present case. The estimation of $\left|c_{21}\right|^{2}$ was made for $R=d_{f} / \sqrt{8 \ln 2}$, where it has the maximum value.

In the region $l<l_{b}$, ionization loss is merely defined by the coherently enhanced contribution from the atomic electron transitions to the continuous spectrum $\left\langle F_{\|}\right\rangle_{\text {cont }}$. The dependence of $d \epsilon / d z$ on $l$ in this region is presently determined by the function $x_{0}(l)$ in Eq. (45). Note that the existence of a series of resonances (instead of a single resonance for each atomic shell) in the region $l>l_{b}$, as well as the ionization loss behavior in the region $l<l_{b}$, defined by coherent effects in atomic ionization, are the novel features of the present more accurate approach compared to the simplified one applied in Ref. [6].

\section{CONCLUSIONS}

In the present paper, ionization loss of ultrarelativistic electron ensembles (bunches) is studied taking into account coherent effects in this loss. Such effects originate from the interference between the electric fields of the electrons and can be manifested for a sufficiently small spatial size of the ensembles. General expressions for the ionization loss in this case are derived with the use of perturbation theory of quantum mechanics. The conditions of applicability of such an approach are thoroughly investigated. Compared to the simplified classical consideration of this problem, reported in Ref. [6], the present approach allows calculating the key parameters defining the ionization loss value. This particularly concerns the effective mean ionization potential of the atomic shell. It is shown that, in the case when coherent effects are manifested, such a potential is defined not just by the structure of the atomic levels, but by the ensemble form factors as well. Numerical calculations are performed for the simplest case which corresponds to the ensemble motion in atomic hydrogen. It is shown that in this case (like in the general case of light or rarefied gases having small plasma frequency) coherent effects can be manifested even under conditions of the full value density effect in the ionization loss. It is demonstrated that, for the ensemble parameters achievable at modern accelerator facilities, coherent effects can lead to an ionization loss increase by several orders of magnitude compared to the result predicted by the Bethe-Bloch formula. Ensembles with different types of longitudinal particle density distribution are considered. It is shown that, in the case of a bunch with a periodical modulation of its density (which is a model of the bunches obtained at free-electron lasers), resonance effects in ionization loss can take place. The fact that coherent effects make the ionization loss depend on the bunch dimensions and shape provides a principle possibility to apply them (like analogous effects in radiation) for problems of diagnostics of charged particle beams.

\section{ACKNOWLEDGMENTS}

The work was partially supported by Projects No. C-2/ 50-2020 and No. F30-2020 of the National Academy of Sciences of Ukraine (budget program "Support for the Development of Priority Areas of Scientific Research," 6541230).

[1] A. E. Chudakov, On an ionization effect associated with observation of electron-positron pairs at very high energy, Izv. Akad. Nauk SSSR 19, 589 (1955).

[2] D. Perkins, Ionization at the origin of electron pairs, and the lifetime of the neutral pion, Philos. Mag. 46, 1146 (1955).

[3] W. Brandt, A. Ratkowski, and R. Ritchie, Energy Loss of Swift Proton Clusters in Solids, Phys. Rev. Lett. 33, 1325 (1974).

[4] W. Brandt and R. Ritchie, Penetration of swift ion clusters through solids, Nucl. Instrum. Methods Phys. Res. 64, 43 (1976).

[5] N. F. Shul'ga and V. V. Syshchenko, Ionization losses of energy of fast clusters being produced during a Coulomb explosion of a molecule and electron-positron pair production in matter, Phys. Lett. A 165, 175 (1992).

[6] S. V. Trofymenko and N. F. Shul'ga, Interference effect in the ionization loss of high-energy electron bunches, Phys. Lett. A 383, 2561 (2019).

[7] V. A. Buts, A. N. Lebedev, and V. I. Kurilko, The Theory of Coherent Radiation by Intense Electron Beams (Springer, Berlin, 2006).

[8] A. P. Potylitsyn, M. I. Ryasanov, M. N. Strikhanov, and A. A. Tishchenko, Diffraction Radiation by Relativistic Particles (Tomsk Polytechnic University, Tomsk, 2008).

[9] Y. Cai, Coherent synchrotron radiation by electrons moving on circular orbits, Phys. Rev. Accel. Beams 20, 064402 (2017). 
[10] E. Fermi, The ionization loss of energy in gases and in condensed materials, Phys. Rev. 57, 485 (1940).

[11] R. M. Sternheimer, M. J. Berger, and S. M. Seltzer, Density effect for the ionization loss of charged particles in various substances,

At. Data Nucl. Data Tables 30, 261 (1984).

[12] V. B. Berestetskii, E. Lifshitz, and L. P. Pitaevskii, Quantum Electrodynamics (Butterworth-Heinemann, Oxford, 2012).

[13] E. L. Duman, L. I. Men'shikov, and B. M. Smirnov, "Destruction" of hydrogen atom by collisions with multiply charged ions, Sov. Phys. JETP 49, 260 (1979), http://www .jetp.ac.ru/cgi-bin/e/index/e/49/2/p260?a=list.

[14] A. B. Voitkiv and A. V. Koval, Excitation and ionization of hydrogen atoms by fast multicharged ions, Sov. Phys. Tech. Phys. 64, 188 (1994), http://journals.ioffe.ru/articles/ 18674.

[15] A. S. Davydov, Quantum Mechanics (Pergamon, Oxford, 1965).

[16] A. I. Akhiezer and N. F. Shul'ga, High Energy Electrodynamics in Matter (Gordon and Breach, Amsterdam, 1996).

[17] L. D. Landau and E. M. Lifshitz, Quantum Mechanics (Pergamon, Oxford, 1965).
[18] Let us note that the same definition of $f_{\|}$and $f_{\perp}$ should be used in Ref. [6]. This requires dividing the values of $l_{f}$ and $d_{f}$ by $\sqrt{2}$ in the figures there. However, it does not make a noticeable change due to the logarithmic scale in the figures.

[19] J. F. Bak, A. Burenkov, J. B. B. Petersen, E. Uggerhøj, S. P. Møller, and P. Siffert, Large departures from Landau distributions for high-energy particles traversing thin $\mathrm{Si}$ and Ge targets, Nucl. Phys. B288, 681 (1987).

[20] J. Zhu, Design study for generating sub-femtosecond to femtosecond electron bunches for advanced accelerator development at SINBAD, Ph. D. thesis, University of Hamburg, Hamburg, 2017, https://bib-pubdb1.desy.de/ record/401420? ln=en.

[21] E. A. Schneidmiller and M. V. Yurkov, Baseline parameters of the European XFEL, in Proceedings of the 38th International Free Electron Laser Conference (FEL 2017), Santa Fe, NM (JACoW, Geneva, 2017), MOP033.

[22] S. Liu, W. Decking, V. Kocharyan, E. Saldin, S. Serkez, R. Shayduk, H. Sinn, and G. Geloni, Preparing for highrepetition rate hard x-ray self-seeding at the European X-ray Free Electron Laser: Challenges and opportunities, Phys. Rev. Accel. Beams 22, 060704 (2019). 